\title{
CENÁRIOS DE NOVOS MODOS DE PRODUÇÃO E CONSUMO NA HABITAÇÃO DE INTERESSE SOCIAL ATRAVÉS DE SISTEMAS PRODUTO+SERVIÇO
}

\section{SCENARIOS OF NEW MODES OF PRODUCTION AND CONSUMPTION IN THE SOCIAL HOUSING THROUGH PRODUCT SERVICE SYSTEMS}

\section{Aline Müller Garcia (UFPR); Aguinaldo dos Santos, Dr. (UFPR)}

\author{
Palavras Chave \\ Hábitos de consumo; Habitação de interesse social; Sistemas produto+serviço; Design para a sustentabilidade.
}

\author{
Key Words \\ Consumption habits; Social housing; Product+service systems; Design for sustainability
}

\section{RESUMO}

Compreender os hábitos e significados do consumo é uma etapa inicial necessária para o desenvolvimento de soluções que promovam mudanças profundas na direção modos de produção e consumo mais sustentáveis. Ao mesmo tempo, o Design de Sistemas Produto+Serviço (PSS) tem se mostrado como uma estratégia promissora no desenvolvimento destes novos modos. Isso acontece na relação direta com o consumidor (B2C) e também nas relações entre empresas (B2B). Assim o objetivo deste artigo é realizar uma análise crítica da literatura acerca do comportamento do morador de Habitação de Interesse Social no Brasil e em outros países emergentes. Esta análise possibilita a identificação de oportunidades latentes para a adoção de soluções em modelos de PSS. A abordagem utilizada é a Revisão Bibliográfica Sistemática (RBS) por meio da qual investigou-se o estado da arte sobre conceitos de PSS, particularmente sobre os estudos voltados a pessoas de baixa renda. Nesta revisão buscou-se também, estudos que tenham avaliado a utilização de PSS como estratégia para influenciar positivamente na mudança de hábitos de consumo. Dessa forma, o artigo procura contribuir com a identificação de oportunidades de inovação orientadas à habitação de interesse social. Sendo assim, o foco é avaliar ofertas que combinam produtos e serviços, levando em conta demandas inferidas a partir da caracterização dos hábitos dos moradores destas habitações.

\section{ABSTRACT}

Understanding the habits and meanings of consumption is a first step for the development of solutions that promote profound changes towards more sustainable modes of production and consumption. At the same time, the Design of Product+Service Systems (PSS) has been shown as a promising strategy in the development of these new modes. This happens in the direct relationship with the consumer (B2C) and also in the relationships between companies (B2B). Thus, the objective of this article is to perform a critical analysis of the literature about the behavior of residents of Social Housing in Brazil and other emerging countries. This analysis allows the identification of latent opportunities for the adoption of solutions in PSS models. The approach used is the Systematic Bibliographic Review, through it, was investigated the state of the art of PSS concepts, particularly on studies aimed at low-income people. In this review also was searched papers that have evaluated the use of PSS as a strategy to positively influence the change in consumption habits. In this way, this paper seeks to contribute to the identification of innovation opportunities directed to Social Housing. Therefore, the focus is to evaluate offers that combine products and services, considering the inferred demands from the characterization of the habits of the residents of these homes. 


\section{INTRODUÇÃO}

Os hábitos de consumo de populações de baixa renda de países emergentes como o Brasil são o foco do presente artigo. Esse estudo é necessário para compreender os hábitos do usuário e desta forma buscar alternativas de consumo mais sustentáveis. Um caminho que tem se mostrado promissor é a mudança dos sistemas tradicionais de consumo, voltados para produção, utilização e descarte de produtos, para conceitos de Sistemas Produto+Serviço (PSS). Manzini e Vezzoli (2002) definem o PSS como resultado de um pensamento estratégico de inovação que muda o foco dos negócios, da simples comercialização de produtos, para sistemas que envolvem produtos e serviços integrados.

Tukker (2004) categorizou três possibilidades para sistemas PSS: "orientado ao produto", "orientado ao uso" e "orientado aos resultados". No PSS "orientado ao produto" o cliente tem a posse do artefato, porém o fabricante e outros stakeholders oferecem serviços de apoio ao ciclo de vida do produto (ex: manutenção ou upgrades). Já no PSS orientado ao uso o cliente tem acesso a uma plataforma de produtos e serviços, não tendo a posse dos produtos envolvidos (ex: plataforma de cozinha compartilhada). Já o PSS "orientado a resultados" é uma modalidade que oferece ao cliente/usuário o resultado final da unidade de satisfação almejada, sem que o cliente/usuário tenha que necessariamente manusear ou operar produtos ou serviços (ex: venda de "superfícies para caminhar" ao invés de carpetes).

Um dos desafios centrais para a disseminação de ofertas de sistemas produto+serviço é a mudança de paradigmas arraigados de produção e consumo. De acordo com Cortez (In: Ortigoza e Cortez, 2009) o consumo está diretamente relacionado com a forma que as pessoas veem o mundo e com o meio social que elas estão inseridas. Impulsionado pelas evoluções tecnológicas e sociais, o ato de consumir, que poderia ser entendido como uma forma controlada e racional de adquirir bens ou produtos para suprir necessidades, tem se configurado como consumismo, ou seja, um modo de consumir para além do que é necessário para suprir as necessidades. $O$ padrão de consumo na sociedade moderna é socialmente injusto, além de ser insustentável para o meio ambiente. Existe uma exploração abusiva dos recursos naturais e a riqueza resultante está longe de ser dividida igualmente para todas as pessoas e apenas uma seleta minoria tem acesso aos benefícios obtidos com os avanços da tecnologia.

A desigualdade social está diretamente relacionada as possibilidades diferentes de acesso aos bens de consumo disponíveis e o Brasil é um dos países onde as diferenças sociais são mais marcantes. Cortez (In: Ortigoza e Cortez,
2009) também fala sobre o problema do desperdício e lista as principais formas de reduzir a demanda por recursos. Em primeiro lugar estão as mudanças de comportamento dos usuários, além disso são citadas alterações na estrutura dos sistemas urbanos e de transporte, ou seja, de um modo geral o comportamento do consumidor e o planejamento de sistemas então entre os principais fatores passiveis de impulsionar mudanças concretas.

A população que está na base da pirâmide econômica e social, ou seja, pessoas que vivem com menos de US\$ 4 por dia, tem despertado o interesse de empresas pois constituem um público muito representativo, principalmente em países emergentes. Os mercados considerados emergentes apresentam uma economia em crescimento, por isso, empresas do mundo todo veem possibilidades de investimentos lucrativos nesses mercados. Somente no BRICS (Brasil, Rússia, Índia, China e África do Sul) que é um grupo político de cooperação econômica de países emergentes, existem aproximadamente três bilhões de pessoas, sendo que a maior parte pertence a base da pirâmide. Porém, nesses países também vivem outra parcela de pessoas que tem um poder de acesso maior aos bens de consumo, que também são considerados um público relevante para investidores (BARKI, BOTELHO e PARENTE, 2013). Assim países como o Brasil estão sendo visados por empresas multinacionais e o consumo tem sido cada vez mais estimulado.

Escolheu-se como método desta pesquisa a Revisão Bibliográfica Sistemática (RBS) partindo do roteiro de Conforto et al. (2011) para a verificação das pesquisas existentes sobre o tema e sob quais vieses elas têm se orientado. Dessa forma, partindo de uma base preliminar sobre Design de PSS, procura-se por meio da RBS entender os hábitos de consumo de pessoas de baixa renda e identificar possíveis cenários para a implantação de PSSs a partir deste estudo. Para possibilitar tal investigação parte-se da seguinte questão: quais são as informações sobre hábitos de consumo em países emergentes e sobre o desenvolvimento de modelos PSS, disponíveis na literatura qualificada? Pretende-se investigar, tanto modelos já existentes de PSS, quanto estudos concentrados nos hábitos de consumo dessas pessoas.

\section{MÉTODO}

Para responder à questão inicial desse artigo escolheu-se como método de investigação a Revisão Bibliográfica Sistemática (RBS). Conforme o roteiro proposto por Conforto et al. (2011) (Figura 01) a RBS possui 15 etapas divididas entre 3 fases. 
Figura 1: Fases e etapas roteiro RBS.

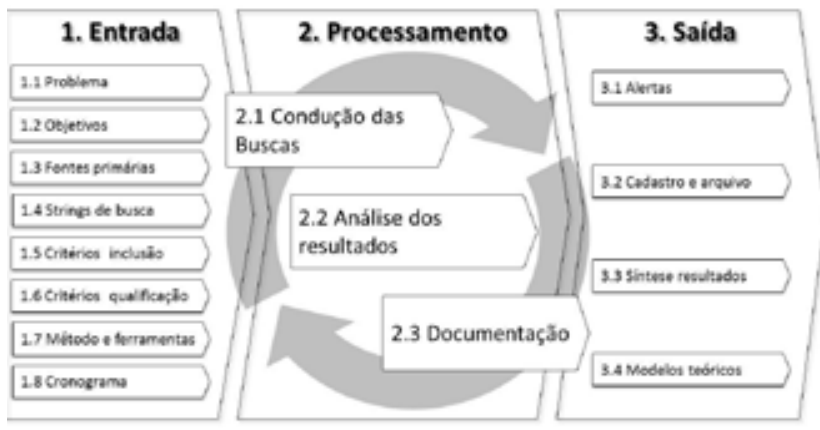

Fonte: Conforto et al. (2011).

Para Conforto et al. (2011) a RBS é o primeiro passo para a elaboração de uma pesquisa científica, pois permite o desenvolvimento de familiaridade com o problema, além de fornecer informações essenciais para verificar-se o ineditismo da pesquisa. Desta forma é possível realizar uma pesquisa bibliográfica sem ênfases na interpretação pessoal, como poderia acontecer em uma revisão narrativa. As informações obtidas nesta RBS são apresentadas neste artigo como resultados iniciais, que serão cruzados com outras etapas, como pesquisa de campo com os usuários para que seja possível validar os dados obtidos.

\subsection{Protocolo da RBS}

Nesta RBS a pergunta de partida foi: quais são as informações sobre hábitos de consumo em países emergentes e sobre o desenvolvimento de modelos PSS disponíveis na literatura qualificada? Identificouse como objetivos a serem alcançados: 1) obter referências bibliográficas e identificar outras pesquisas que abordem PSS e hábitos de consumo e pessoas de baixa renda; 2) verificar possibilidades de abertura e disponibilidade para aceitar modelos mais desmaterializados de consumo. Para caracterizar o perfil do problema sob o ponto de vista do nível de consolidação do conhecimento no tema realizou-se inicialmente um levantamento bibliométrico na Biblioteca Digital de Teses e Dissertações (BDTD). Foram pesquisados trabalhos (dissertações e teses) realizados entre os anos de 2009 a 2018, sendo o resultado desta busca sintetizado no Quadro 01 a seguir:
Quadro 1: Teses e Dissertações entre 2009 e 2018 em temas associados à dissertação.

\begin{tabular}{|c|c|}
\hline PALAVRAS-CHAVE & TOIAL \\
\hline "Matito de tomar banbo" & a. \\
\hline "Contumo sustentivel" ARUD design & 12 \\
\hline "Consumo anstentivet" AND "habitaģäs de interesse social" & 1 \\
\hline "Consume sustentivert" AND "Sistemas Produto+5ervige" & 2 \\
\hline "Uso racional da agua e energia" AND Thabitaçálo de interesse social" & 0 \\
\hline "Uso racional da igua e enerigia" AND "Sistemas Produto+Serviço" & 0 \\
\hline "Ststemas Produto*Servico" AND "habitacso de interesse social" & 3 \\
\hline TOTAL SEM REPETIDOS & 15 \\
\hline
\end{tabular}

Fonte: Elaborado pelos autores.

Do total de trabalhos identificados, seis foram realizadas em programas de pós-graduação em Design ecinco em programas de pós-graduação em Ciência, Tecnologia e Sociedade. Todos os seis trabalhos provenientes do campo do Design foram desenvolvidos no PPGDesign da UFPR no qual esta pesquisa esta também inserida. Destes trabalhos cinco deles são fundamentados em pesquisas que envolvem empresas, tecnologia e desenvolvimento (GIGANTE, 2012; YANAl, 2012; BESKOW, 2013; TUCCl, 2012; GOMES, 2011); a questão do consumo sustentável está presente em quatro dos trabalhos selecionados (GOMES, 2009; TEIXEIRA, 2011; DAROS, 2013; SANTOS, 2013); o Design de Sistemas Produto+Serviço também é o tema de quatro dos trabalhos encontrados (COSTA JUNIOR, 2012; FONTANA, 2013; DAROS, 2013; HOSS, 2014).

Pesquisas que envolvem pessoas de baixa renda e habitação de interesse social também surgiram como resultados desta busca (COSTA JUNIOR, 2012; DAROS, 2013; HOSS, 2014; CACCERE, 2017), sendo que a questão da educação associada ao tema foi identificada em três dissertações (GOMES, 2009; COSTA JUNIOR, 2012; MEZZACAPPA, 2013). Neste contexto, entende-se que as interfaces entre os temas "consumo sustentável", "uso racional da água e energia" e "sistema produto+serviço", enfatizando a habitação de interesse social, configura em relativa lacuna do conhecimento no campo do Design no Brasil.

Outra pesquisa bibliométrica foi realizada com foco em periódicos utilizando o protocolo proposto por Conforto et al. (2011), tendo sido realizada nos meses de agosto e setembro de 2017, sendo que os critérios de inclusão foram artigos posteriores ao ano de 2007,avaliados por pares e produzidos no idioma inglês. Para um primeiro filtro realizou-se a leitura dos títulos, palavras-chave e resumo dos artigos. $O$ segundo filtro estende-se a leitura para a introdução e conclusão se necessário. Finalmente, para o terceiro filtro foi realizado a leitura completa dos artigos e o fichamento dos que foram selecionados. Os resultados desta busca são mostrados no Quadro 02 a seguir. 
Quadro 2: Relação dos resultados da RBS.

\begin{tabular}{|c|c|c|c|c|c|c|c|}
\hline STRINGS & CAPES & SCOPUS & BDTD & DUPLICADOS & FILTR0 01 & FILTRO02 & FILTR0 03 \\
\hline $\begin{array}{l}\text { "consumption habits" AND } \\
\text { "social interest housing" }\end{array}$ & 1 & 0 & 2 & 0 & 3 & 0 & 0 \\
\hline $\begin{array}{l}\text { "product service systems" } \\
\text { AND"social interest housing" }\end{array}$ & 0 & 0 & 1 & 0 & 1 & 1 & 1 \\
\hline $\begin{array}{l}\text { "consumption habits" AND } \\
\text { "bottom of the pyramid" }\end{array}$ & 27 & 0 & 1 & 2 & 26 & 3 & 1 \\
\hline $\begin{array}{l}\text { "consumption habits" AND } \\
\text { "bottom of the pyramid" }\end{array}$ & 21 & 2 & 0 & 2 & 19 & 2 & 0 \\
\hline $\begin{array}{l}\text { "consumption habits" } \\
\text { AND "low income" }\end{array}$ & 291 & 9 & 14 & 16 & 275 & 41 & 11 \\
\hline $\begin{array}{l}\text { "product service systems" } \\
\text { AND "low income" }\end{array}$ & 14 & 2 & 0 & 2 & 14 & 3 & 1 \\
\hline $\begin{array}{l}\text { "sustainable consumption" } \\
\text { AND "service design" }\end{array}$ & 50 & 4 & 0 & 4 & so & 9 & 2 \\
\hline $\begin{array}{l}\text { "sustainable consumption" } \\
\text { AND "product service } \\
\text { systems" }\end{array}$ & 137 & 5 & 2 & 5 & 139 & 27 & 8 \\
\hline $\begin{array}{l}\text { "sustainable consumption" } \\
\text { AND "low income" }\end{array}$ & 364 & 0 & 0 & 0 & 364 & 89 & 20 \\
\hline \multicolumn{5}{|c|}{ RESULTADOS } & 891 & 175 & 44 \\
\hline
\end{tabular}

Fonte: Elaborado pelos autores 
A partir da leitura dos 44 trabalhos selecionados para a RBS elaborou-se um quadro para ilustrar os direcionamentos das pesquisas mais relevantes para esta pesquisa. O Quadro 3 apresenta os resultados referentes ao consumo e comportamento em países emergentes:

Quadro 3: Comparativo entre os artigos analisados.

\begin{tabular}{|c|c|c|c|c|}
\hline Artigos & Objetivos & $\begin{array}{l}\text { Unidade de } \\
\text { Satisfação }\end{array}$ & Método & $\begin{array}{l}\text { País emergente } \\
\text { (onde a pesquisa foi realizada) }\end{array}$ \\
\hline $\begin{array}{l}\text { Shäfer, Jaeger- } \\
\text { Erben e Santos } \\
(2010)\end{array}$ & $\begin{array}{l}\text { Caracterizar } \\
\text { consumidores }\end{array}$ & Alimentação & Survey & Brasil \\
\hline $\begin{array}{l}\text { Sheth, Sethia e } \\
\text { Srinivas (2010) }\end{array}$ & $\begin{array}{l}\text { Promover aborda- } \\
\text { gem centrada no } \\
\text { usuário }\end{array}$ & - & $\begin{array}{l}\text { Revisão de } \\
\text { Literatura }\end{array}$ & - \\
\hline $\begin{array}{l}\text { Barbosa e Veloso } \\
(2012)\end{array}$ & $\begin{array}{l}\text { Caracterizar motiva- } \\
\text { ções de consumo }\end{array}$ & $\begin{array}{l}\text { Alimentação e } \\
\text { Higiene pessoal }\end{array}$ & Estudo de Caso & Brasil \\
\hline $\begin{array}{l}\text { Chickweche, } \\
\text { Stanton e Fletcher } \\
(2012)\end{array}$ & $\begin{array}{l}\text { Decisão de compra } \\
\text { na base da pirâmide }\end{array}$ & - & Estudo de Caso & Zimbabwe \\
\hline $\begin{array}{l}\text { Carrete et al. } \\
(2012)\end{array}$ & $\begin{array}{l}\text { Caracterizar motiva- } \\
\text { ções de consumo }\end{array}$ & - & Estudo de caso & México \\
\hline $\begin{array}{l}\text { Forcato e Santos } \\
(2015)\end{array}$ & $\begin{array}{l}\text { Estratégias para } \\
\text { promover compor- } \\
\text { tamento sustentável }\end{array}$ & Lavagem de roupas & Estudo de caso & Brasil \\
\hline $\begin{array}{l}\text { O'Rourke e Lollo } \\
\text { (2015) }\end{array}$ & $\begin{array}{l}\text { Caracterizar motiva- } \\
\text { ções de consumo }\end{array}$ & $\begin{array}{l}\text { Transporte; } \\
\text { energia; } \\
\text { alimentação e } \\
\text { habitação }\end{array}$ & $\begin{array}{l}\text { Revisão de } \\
\text { Literatura }\end{array}$ & China \\
\hline
\end{tabular}

Fonte: Elaborado pelos autores 
A maior parte dos trabalhos verificados procuram caracterizar comportamentos e motivações de consumo como os artigos de Shäfer, Jaeger-Erben e Santos (2010), Barbosa e Veloso (2012), Carrete et al. (2012) e O'Rourke e Lollo (2015). Alimentação, uma unidade de satisfação para qual muitas pesquisas estão voltadas, seguida por abastecimento de energia e água. Estudos de casos são as abordagens mais utilizadas. Trabalhos realizados no contexto do Brasil foram priorizados, porém outros trabalhos com dados importantes sobre outros países de economia emergente como México, China e África também foram considerados.

Quanto os trabalhos que descrevem serviços ou PSS para contextos emergentes, os 9 trabalhos selecionados (todos artigos) também foram agrupados no Quadro 4 para ilustrar e possibilitar a discussão desses dados.

Quadro 4: Comparativo entre os artigos analisadas.

\begin{tabular}{|c|c|c|c|c|}
\hline Artigos & Objetivos & $\begin{array}{l}\text { Unidade de } \\
\text { Satisfação }\end{array}$ & Método & País emergente \\
\hline $\begin{array}{l}\text { Vezzoli e Sciama } \\
\text { (2007) }\end{array}$ & $\begin{array}{l}\text { Capacitar estudantes deDesign } \\
\text { para desenvolver soluções susten- } \\
\text { táveis para contextos emergentes. }\end{array}$ & Ensino & $\begin{array}{l}\text { Ensino } \\
\text { colaborativo }\end{array}$ & $\begin{array}{l}\text { Brasil, Índia e } \\
\text { China } \\
\text { (Inicialmente) }\end{array}$ \\
\hline $\begin{array}{l}\text { Hall e Lobina } \\
\text { (2007) }\end{array}$ & $\begin{array}{l}\text { Verificar possibilidades do setor } \\
\text { privado oferecer serviços básicos. }\end{array}$ & $\begin{array}{l}\text { Fornecimento de } \\
\text { água e saneamento }\end{array}$ & Estudo de caso & $\begin{array}{l}\text { Países da América } \\
\text { Latina e da África }\end{array}$ \\
\hline $\begin{array}{l}\text { Fernández- } \\
\text { Maldonado } \\
\text { (2008) }\end{array}$ & $\begin{array}{l}\text { Analisar casos de serviços básicos } \\
\text { oferecidos pelo setor privado. }\end{array}$ & $\begin{array}{l}\text { Fornecimento de } \\
\text { água e } \\
\text { telecomunicações }\end{array}$ & Estudo de caso & Peru \\
\hline $\begin{array}{l}\text { Boradkar e } \\
\text { Kilkarni (2010) }\end{array}$ & $\begin{array}{l}\text { Apresentar potencias econômicos } \\
\text { dos mercados emergentes. }\end{array}$ & & \begin{tabular}{|l|} 
Revisão \\
Bibliográfica
\end{tabular} & Índia \\
\hline $\begin{array}{l}\text { Shafer, Parks e } \\
\text { Rai (2011) }\end{array}$ & $\begin{array}{l}\text { Propor caminhos para o desen- } \\
\text { volvimento sustentável em países } \\
\text { emergentes. }\end{array}$ & & Estudo de caso & \\
\hline $\begin{array}{l}\text { Shäfer, Kebir e } \\
\text { Neumann (2011) }\end{array}$ & $\begin{array}{l}\text { Mostrar os desafios para o forne- } \\
\text { cimento de energia em contextos } \\
\text { de baixa renda. }\end{array}$ & $\begin{array}{l}\text { Fornecimento de } \\
\text { energia }\end{array}$ & $\begin{array}{l}\text { Revisão } \\
\text { Bibliográfica }\end{array}$ & \\
\hline $\begin{array}{l}\text { Friebe, Flotow e } \\
\text { Täube (2013) }\end{array}$ & $\begin{array}{l}\text { Quantificar os principais elemen- } \\
\text { tos em PSS para contextos de } \\
\text { baixa renda. }\end{array}$ & Energia solar & $\begin{array}{l}\text { Revisão } \\
\text { Bibliográfica e } \\
\text { Estudo de caso }\end{array}$ & Índia \\
\hline $\begin{array}{l}\text { Ceschin e } \\
\text { Gaziulusoy } \\
\text { (2016) }\end{array}$ & $\begin{array}{l}\text { Analisar a evolução do Design } \\
\text { para a sustentabilidade nas últi- } \\
\text { mas décadas. }\end{array}$ & & $\begin{array}{l}\text { Revisão } \\
\text { Bibliográfica }\end{array}$ & \\
\hline $\begin{array}{l}\text { Emile, Ceschin e } \\
\text { Harrison (2016) }\end{array}$ & $\begin{array}{l}\text { Identificar e validar modelos para } \\
\text { fornecimento de energia em paí- } \\
\text { ses emergentes. }\end{array}$ & $\begin{array}{l}\text { Fornecimento de } \\
\text { energia }\end{array}$ & Estudo de Caso & $\begin{array}{l}\text { Botswana e África } \\
\text { do Sul }\end{array}$ \\
\hline
\end{tabular}

Fonte: Elaborado pelos autores.

Por meio do quadro comparativo percebe-se que a abordagem dos trabalhos selecionados variam desde comparações entre serviços públicos e privados e seus impactos na sociedade de baixa renda, até estudo e aplicação de estratégias para o desenvolvimento sustentável, como os PSSs.

Quanto aos hábitos de consumo foram identificadas muitas informações relevantes para este artigo. Por exemplo, Carrete et. al (2012) concluíram que independente da condição social ou localização geográfica, os consumidores mexicanos não possuíam muita preocupação com o meio ambiente. Quanto a reciclagem do lixo, não houve diferença nas pesquisas entre usuários com mais ou menos recursos financeiros; sobre o consumo de alimentos orgânicos, regiões no norte do México (influenciadas pelo estilo de vida americano) não os consumiam 
mais que em outras partes do país. Entretanto as famílias mexicanas procuravam economizar energia e água, porém essa iniciativa estava mais relacionada a economia nas respectivas tarifas do que propriamente em iniciativas sustáveis. Para Daros (2013) em seu estudo com moradores de HIS, os hábitos de consumo são influenciados por aspectos sociais, culturais, ambientais e do contexto. A autora argumenta que os consumidores focam na utilização dos produtos de modo que recursos envolvidos (como água e energia) tornam-se invisíveis e acabam por ser esquecidos. Outros fator é a impossibilidade de medir em tempo real a quantidade de água ou energia que cada produto consome. A autora também cita negligência, percepção de que atitudes individuais não fazem diferença, falta de confiança e estilos de vida influenciados pelo consumismo.

Os campos mais explorados dentre os artigos encontrados são o fornecimento de água e energia, sendo que também aparece um artigo com estratégias para o ensino de estudantes de design. Outro artigo, além do fornecimento de energia, também aborda as telecomunicações. Revisões bibliográficas seguidas por estudos de caso tem sido os métodos mais efetivos para elucidar as discussões e soluções propostas nos trabalhos. Quanto aos países emergentes onde estão sendo realizadas as pesquisas na área encontram-se casos no Brasil, outros países da América Latina, Índia e países da África.

\section{CONCLUSÃO}

As informações obtidas por meio da realização desta revisão apontam a direção dos trabalhos realizados na área de desenvolvimento de PSSs para população de baixa renda a partir de seus hábitos de consumo. Considerouse dentre os resultados, tanto pesquisas voltadas a compreender os padrões de consumo em países emergentes, como o Brasil, quanto estratégias de inovação sustentável para desenvolvimento desses locais. Muitos estudos sobre PSS tem-se voltado aos mercados emergentes com a intenção de possibilitar desenvolvimento nos três níveis: econômico, social e ambiental.

No que diz respeito aos estudos sobre comportamento dos consumidores, muitas pesquisas voltam-se a caracterizar o consumidor e seus padrões de consumo. O foco tem sido procurar caminhos para solucionar primeiro as necessidades básicas das pessoas, por isso alimentação, fornecimento de água e energia tem sido temas recorrentes, mas de fundamental importância no contexto de populações de baixa renda. Quanto aos resultados referentes a criação de serviços para essa população, os estudos procuram direcionar oportunidades e desafios para solucionar problemas sociais de forma sustentável.

Assim, é possível identificar, a partir das informações encontradas nos materiais consultados, um panorama das pesquisas voltadas à criação de produtos e serviços para contextos emergentes. O campo de trabalho é bastante amplo, sendo que as necessidades desses usuários são urgentes em todos os níveis. Verifica-se que soluções que envolvem desenvolvimento de PSSs tem sido utilizadas e podem ser efetivas, desde que os usuários sejam envolvidos no processo de desenvolvimento e implementação e sejam ensinados e constantemente lembrados da importância da utilização consciente dos recursos.

Dessa forma, os objetivos propostos para este artigo foram alcançados, sendo que foi possível obter referências bibliográficas sobre hábitos de consumo e PSS para populações de baixa renda e verificar que um caminho para a aceitação desse conceito é a educação e a participação do usuário. Assim reuniu-se material para responder a questão inicial e dar base teórica para o desenvolvimento de pesquisas que procurem atuar nestes contextos.

\section{AGRADECIMENTOS}

Os autores agradecem o apoio financeiro da CAPES (Coordenação de Aperfeiçoamento de Pessoal de Nível Superior) com a bolsa de Mestrado do curso de PósGraduação em Design pela Universidade Federal do Paraná.

\section{REFERÊNCIAS}

BARBOSA, Livia; VELOSO, Leticia. Consumption, domestic life and sustainability in Brazil. Journal of Cleaner Production, Elsevier, 63, 2014. p. 166 - 172.

BARKI, Edgard; BOTELHO, Delane; PARENTE, Juracy. Varejo: desafios e oportunidades em mercados emergentes. Revista de Administração de Empresas, v. 53, n. 6, p. 534-538, 2013.

BORADKAR, Prasad; KULKARNI, Unmesh. Design Tools for Base of the Pyramid Strategies. Interactions, 17, novembro - dezembro, 2010. p. 41 - 46.

CARRETE, Lorena. et. al. Green consumer behavior in an emerging economy: confusion, credibility, and compatibility. Journal of Consumer Marketing, Vol. 29, 7, 2012. p. $470-481$. 
CESCHIN, Fabrizio; GAZIULUSOY, Idil. Evolution of design for sustainability: From product design to design for system innovations and transitions. Design Studies, Elsevier, Vol. 47, novembro, 2016. p. 118 - 163.

CHICKWECH, Tendai; STANTON, John; FLETCHER, Richard. Family purchase decision making at the bottom of the pyramid. Journal of Consumer Marketing, Emerald Insight, Vol. 29, 3. p. 202 - 213.

CONFORTO, Edivandro Carlos. et al. Roteiro para revisão bibliográfica sistemática: aplicação no desenvolvimento de produtos e gerenciamento de projetos. In: $8^{\circ}$ CONGRESSO BRASILEIRO DE GESTÁO DE DESENVOLVIMENTO DE PRODUTOS - CBGDP. Anais p. 1 - 12, Porto Alegre, 2011.

DAROS, Carolina. Design para a sustentabilidade: oportunidades de inovação a partir dos hábitos de consumo na Habitação de Interesse Social. $n^{\circ} 182$ f. Dissertação (Mestrado em Design). - Universidade Federal do Paraná - UFPR, Curitiba, 2014.

EMILI, Silvia; CESCHIN, Fabrizio; Harrison, David. Product-Service System applied to Distributed Renewable Energy: A classification system, 15 archetypal models and a strategic design tool. Energy for Sustainable Development, Elsevier, 32, 2016. p. 71 - 98

FERNÁNDEZ-MALDONADO, Ana María. Expanding networks for the urban poor: Water and telecommunications services in Lima, Peru. Geoforum, Elsevier, 39, 2008. p. $1884-1896$

FERREIRA, Hugo Chaves Barreto. A insustentável leveza do ter: consumismo como externalidade negativa do Brasil pós-abertura comercial (1990 - 2008). n 104 f. Dissertação (Mestrado em Design). - Universidade Federal de Pernambuco - UFP, Recife, 2009.

FORCATO, Marcelo dos Santos; SANTOS, Aguinaldo dos. O eco-feedback na interface da lavadora de roupas como estratégia de design para a mudança de comportamento. infodesign, São Paulo, v. 12, n.1, 2015. p. 77 - 92

FORCATO, Marcelo dos Santos. Design para o comportamento sustentável: estudo da aplicação do eco-feedback na interface da lavadora de roupas. $n^{\circ} 199$ f. Dissertação (Mestrado em Design). - Universidade Federal do Paraná
- UFPR, Curitiba, 2014.

FRIEBE, Christian A.; VON FLOTOW, Paschen; TÄUBE, Florian A. Exploring the link between products and services in low-income marketsEvidence from solar home systems. Energy Policy, Elsevier, 52, 2013. p. 760 - 769

HALL, David; LOBINA; Emanuele. ProWtability and the poor: Corporate strategies, innovation and sustainability. Geoforum, Elsevier, 38, 2008. p. 772 - 785

HOSS, Maurício Junior. Prototipagem de serviços: um estudo exploratório com foco na iluminação de habitações de interesse social. $n^{\circ} 182$ f. Dissertação (Mestrado em Design). - Universidade Federal do Paraná - UFPR, Curitiba, 2014.

MANZINI, Ezio; VEZZOLI, Carlo. O desenvolvimento de produtos sustentáveis: os requisitos ambientais dos produtos industriais. Edusp, São Paulo, 2002

O'ROURKE, Dara; LOLLO; Niklas. Transforming Consumption: From Decoupling, to Behavior Change, to System Changes for Sustainable Consumption. Annual Review of Environment and Resources, vol. 40, 2015. p. $233-259$

ORTIGOZA, Silvia Aparecida G; CORTEZ, Ana Tereza C. Da produção ao consumo: impactos socioambientais no espaço urbano. UNESP; São Paulo: Cultura Acadêmica, 2009. 146 p.

RIBEIRO, Marília Ferraz. Inclusão social dos usuários de baixa renda por meio do sistema de aquecimento solar. $n^{\circ} 217$ f. Dissertação (Mestrado em Design). Universidade Estadual de Campinas, Faculdade de Eng. Civil, Arquitetura e Urbanismo, Campinas, 2010

SALDANHA, Carlos. Os consumidores das camadas de baixa renda e o valor percebido de seu consumo: uma abordagem qualitativa. $n^{\circ} 97$ f. Dissertação (Mestrado em Design). - Universidade Federal do Rio Grande do Sul - UFRGS, Porto Alegre, 2013

SAMPAIO NETO, José Vianna. A compra do botijão de gás na cidade de Petrópolis (RJ): uma tradição de família. $n^{\circ} 107$ f. Dissertação (Mestrado em Design). - Escola Brasileira de Administração Pública e de Empresas, Centro de Formação Acadêmica e Pesquisa. Rio de Janeiro, 2014 
SCHÃFER, Martina; JAEGER-ERBEN, Melanie; SANTOS, Aguinaldo dos. Leapfrogging to Sustainable Consumption? An Explorative Survey of Consumption Habits and Orientations in Southern Brazil. Journal of Consumer Policy, Econpapers, vol. 34, 2011. p. 175 - 196

SCHÃFER, Martina; KEBIR, Noara; NEUMANN, Kirsten. Research needs for meeting the challenge of decentralized energy supply in developing countries. Energy for Sustainable Development, Elsevier, 15, 2011. p. 324 - 329

SCHAFER, Carson; PARKS, Richard; RAI, Rahul. Design for emerging bottom of the pyramid markets: A Product Service System (PSS) based approach. In: INTERNATIONAL DESIGN ENGINEERING TECHNICAL CONFERENCES \& COMPUTERS ANS INFORMATION IN ENGINEERING CONFERENCE, 2011, Washington, 23 International Conference on Design Theory and Methodology; $16^{\circ}$ Design for Manufacturing and the Life Cycle Conference, Washington: ASME, 2011. p. 28-31

SERBENA, Henrique José. Plataforma de luminária LED para habitação de interesse social. n 201 f. Dissertação (Mestrado em Design). - Universidade Federal do Paraná - UFPR, Curitiba, 2013.

SHETH, Jagdish N.; SETIA, Nirmal K.; SHANTHI, Srinivas. Mindful consumption: a customer-centric approach to sustainability. Journal of the Acadademic Marketing Science, 39, 2011. p. $21-39$

TUKKER, Arnold. Eight types of product-service system: eight ways to sustainability? Experiences from SusProNet. Business Strategy and the Environment, 13, 2004. p. $246-260$

VEZZOLI, Carlo; SCIAMA, Dalia Experimental educational networking on open research issues: Studying PSS applicability and development in emerging contexts. International Journal of Sustainability in Higher Education, vol. 8, 2, 2007. p. $198-209$ 